\title{
OPERATIONAL IMPLEMENTATION OF VARIATIONAL DATA ASSIMILATION
}

\author{
PIERRE GAUTHIER \\ Meteorological Service of Canada \\ Dorval, Québec, Canada
}

\section{Introduction}

Over the last few years, the variational form of statistical estimation has been implemented at many operational centres. The motivation originated from the difficulties associated with the assimilation of satellite data such as TOVS (TIROS-N Operational Vertical Sounders) radiances. Lorenc (1986) showed that the statistical estimation problem could be cast in a variational form (3D-Var) which is just a different way of solving the problem that the so-called optimal interpolation attempts to solve directly. Eyre (1989) showed, in a 1D-Var context, that a variational formulation leads to a more natural framework for the direct assimilation of radiances instead of retrieved temperature and humidity profiles. This is also true for any indirect measurement of the state of the atmosphere. Talagrand and Courtier (1987) showed that the use of the adjoint of a numerical model makes it possible to determine the initial conditions leading to a forecast that would best fit data available over a finite time interval. These two formulations can be combined to yield what is now called the 4D variational formulation of the statistical estimation problem or $4 \mathrm{D}$-Var.

The first implementation of 3D-Var was done at NCEP (Parrish and Derber, 1992) and later on at ECMWF (Courtier et al., 1998). Other centres like the Canadian Meteorological Centre (Gauthier et al., 1999) and the Met Office (Lorenc et al., 2000) also implemented operationally a 3D-Var scheme. Courtier (1997) noted that there exists a dual formulation of 3D-Var on which is based the assimilation of NASA's Data Assimilation Office (Cohn et al., 1998) and of the US Naval Research Laboratory (Daley and Barker, 2000). In 1997, ECMWF implemented 4D-Var (Rabier et al., 2000) and so did Météo-France in 2000 (Gauthier and Thépaut, 2001). A considerable amount of research was necessary to achieve these operational implementations. Courtier et al. (1994) pointed out that a direct implementation of 4D-Var requires a computational time exceeding the capacity of even the most powerful computers. The incremental formulation of 4D-Var was proposed in which a simplified model is used to perform inner iterations followed by an integration of the full model based on the updated initial conditions. This outer iteration provides an updated evaluation of the innovations and of the reference trajectory required to define the simplified tangent linear model. In this context, an operational implementation of 4D-Var is possible. In recent years, experimentation with now operational 4D-Var systems indicates that it is necessary to 
make the simplified model to agree more closely with the complete high-resolution model both for its dynamics and physical parameterizations. This question is the object of current research regarding the nature of the simplified physical parameterizations that need to be used (Janisková et al., 1999; Mahfouf, 1999).

In the context of the data assimilation cycles, the background error statistics should reflect the information gained from past observations, which is implicitly contained in the background state. Fisher and Andersson (2001) report recent results of experiments with a reduced rank Kalman filter, used to provide flow dependent background error covariances to $4 \mathrm{D}$-Var. Their results did not show substantial improvements in the forecasts. This topic is being investigated using different types of simplified Kalman filters.

In this paper, the focus will be on the variational formulation of the data assimilation problem. In section 2, the incremental formulation will be presented and discussed first in the context of 3D-Var. Section 3 presents the incremental form of 4D-Var. Results with a simple barotropic model are presented to illustrate the capabilities and limitations of the approach. Section 4 reviews some recent results obtained from experimentations by several groups (e.g., ECMWF, Météo-France). Section 5 discusses some avenues being explored in current research. This includes the ensemble Kalman filter (Evensen, 1997; Houtekamer and Mitchell, 2001) or representer algorithms (Bennett and Thorburn, 1982; Xu and Daley, 2002).

\section{The incremental formulation of variational assimilation}

The variational assimilation problem is expressed here as

$$
J(\boldsymbol{X})=\frac{1}{2}\left(\boldsymbol{X}-\boldsymbol{X}_{b}\right)^{T} \boldsymbol{B}^{-1}\left(\boldsymbol{X}-\boldsymbol{X}_{b}\right)+\frac{1}{2}(\boldsymbol{H}(\boldsymbol{X})-\boldsymbol{y})^{T} \boldsymbol{R}^{-1}(\boldsymbol{H}(\boldsymbol{X})-\boldsymbol{y})
$$

where $\boldsymbol{X}$ is the model state, $\boldsymbol{X}_{b}$ is the background state, $\boldsymbol{B}$ represents the backgrounderror covariances, $\boldsymbol{y}$ is the observation vector, $\boldsymbol{H}$, the observation operator while $\boldsymbol{R}$ represents the observation error covariances. To precondition the minimization, the

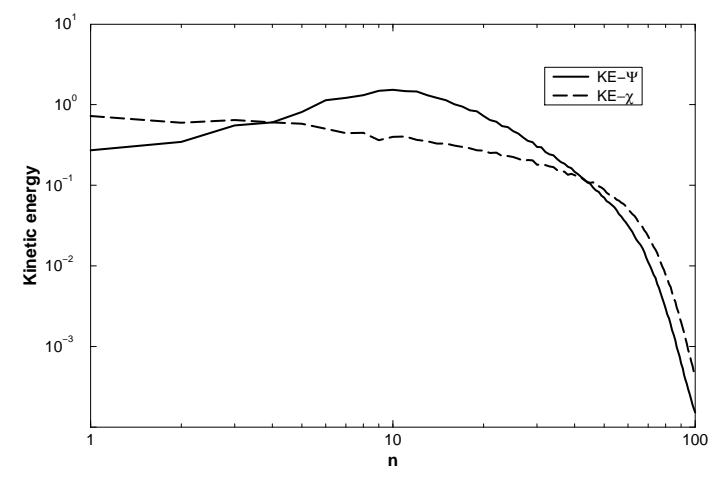

Figure 1. Kinetic energy spectra for the rotational and divergent component for the autocorrelation of background-error statistics at $500 \mathrm{hPa}$. 
control variable $\xi=\boldsymbol{B}^{-1 / 2}\left(\boldsymbol{X}-\boldsymbol{X}_{b}\right)$ is introduced so that $\boldsymbol{X} \equiv \boldsymbol{X}(\xi)=\boldsymbol{X}_{b}+\boldsymbol{B}^{1 / 2} \xi$ and (2.1) can be rewritten as

$$
J(\xi)=\frac{1}{2} \xi^{T} \xi+\frac{1}{2}(\boldsymbol{H}(\boldsymbol{X}(\xi))-\boldsymbol{y})^{T} \boldsymbol{R}^{-1}(\boldsymbol{H}(\boldsymbol{X}(\xi))-\boldsymbol{y}) .
$$

In 3D-Var, the analysis increment $\delta \boldsymbol{x}=\boldsymbol{B}^{1 / 2} \xi$ has an effective lower resolution that is dictated by the background-error covariances. For example, Fig.1 shows the spectrum of the rotational and divergent kinetic energy of correlations. The use of such covariances will lead to an analysis increment with a resolution that cannot exceed 200 $\mathrm{km}$.

(a) Analysis increments from control

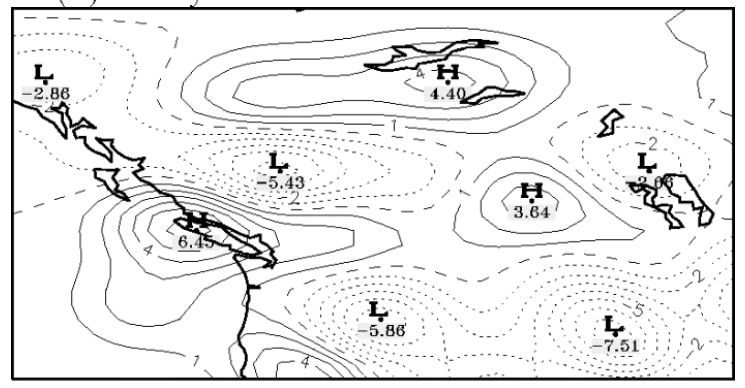

(b) Control - Incremental

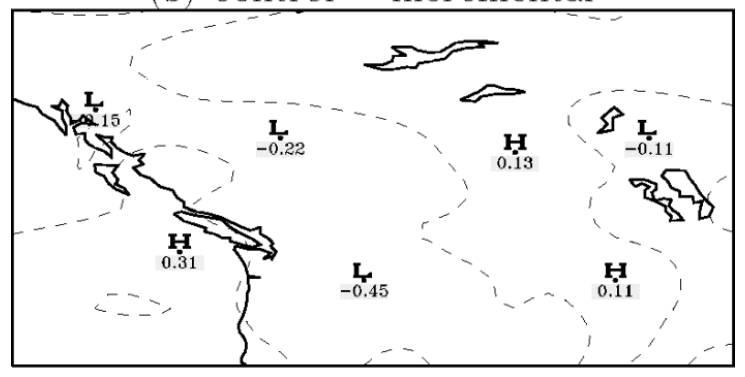

(c) Control - Non_incremental

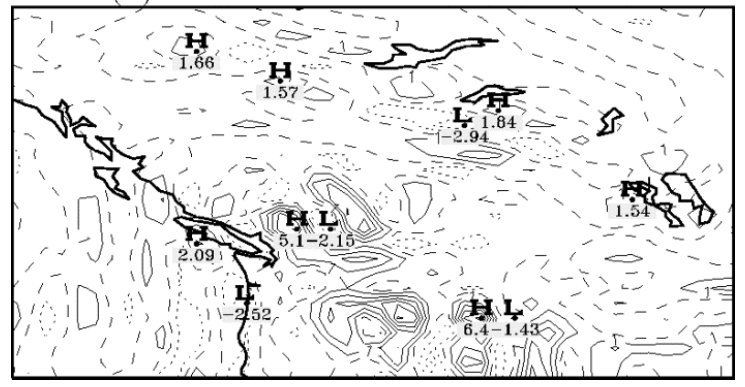

Figure 2. a) Analysis increments of dew point depression at $700 \mathrm{hPa}$ from the control experiment valid at 1200 UTC 24 September 1997. b) Analysis increment of a) minus that of the incremental experiment. c). Analysis increments shown in a) minus that of the non-incremental experiment (from Laroche et al., 1999) 
However, it is beneficial to compute the innovations $\boldsymbol{y}^{\prime}=\boldsymbol{y}-\boldsymbol{H}\left(\boldsymbol{X}_{b}\right)$ using the background state at its full resolution. Following Courtier et al. (1994), we approximate $\boldsymbol{H}(\boldsymbol{X}) \cong \boldsymbol{H}\left(\boldsymbol{X}_{b}\right)+(\partial \boldsymbol{H} / \partial \mathbf{X})\left(\boldsymbol{X}_{b}\right) \delta \mathbf{x} \equiv \boldsymbol{H}\left(\boldsymbol{X}_{b}\right)+\boldsymbol{H}^{\prime}\left(\boldsymbol{X}_{b}\right) \delta \mathbf{x}$ so that (2.2) is now approximated by

$$
J_{L}(\xi)=\frac{1}{2} \xi^{T} \xi+\frac{1}{2}\left(H^{\prime}\left(\boldsymbol{X}_{b}\right) \delta \boldsymbol{x}(\xi)-\boldsymbol{y}^{\prime}\right)^{T} \boldsymbol{R}^{-1}\left(\boldsymbol{H}^{\prime}\left(\boldsymbol{X}_{b}\right) \delta \boldsymbol{x}(\xi)-\boldsymbol{y}^{\prime}\right)
$$

with $\delta \boldsymbol{x}(\xi)=\boldsymbol{B}^{1 / 2} \xi$. As pointed out in Courtier et al. (1994), $J_{L}$ has a form very similar to the original problem except that the observation operator has been linearized around $\boldsymbol{X}_{b}$ and the resulting Jacobian, $\boldsymbol{H}^{\prime}\left(\boldsymbol{X}_{b}\right)$, is used instead of the nonlinear form of the observation operator. Fig.2 from Laroche et al. (1999) shows the analysis increment obtained by using the original cost function at the full resolution of the model and differences between this analysis increment and that obtained by using (2.3) (Fig.2b). The differences indicate that the analyses are virtually identical. Fig.2c however shows the difference between the reference analysis increment and that obtained by solving (2.1) but at a lower resolution. The differences are significant and stress the importance of computing the innovations with respect to the full resolution of the background field.

This approach has been used at the Canadian Meteorological Centre (Laroche et al., 1999) and Météo-France (Desroziers et al., 2003) to produce regional analyses for a variable resolution model while the analysis increment remains global and at a coarser resolution. The small scales features found in the analysis are therefore produced by the model itself in response to changes brought by the analysis to the large-scale components.

\section{The incremental form of $4 \mathrm{D}-$ Var}

The reasons why low-resolution increments are sufficient in 4D-Var are different than those presented above for 3D-Var. In Thépaut and Courtier (1991) and later on in Tanguay et al. (1995) and Laroche and Gauthier (1999), it is shown that for the largescale dynamics, 4D-Var adjusts the more energetic large-scale components first. To determine the analysis increments of 4D-Var within the inner loop, Courtier et al. (1994) then proposed to use the tangent linear model (TLM) and its adjoint (Adj) at a coarser resolution with simplified physical parameterizations. However, as for the 3DVar case, the innovations must be computed with respect to a high-resolution trajectory generated by the high-resolution model with its complete set of physical parameterizations. Outer loops are however needed to update the reference trajectory that defines the TLM and Adj.

Two questions are then raised. First, is it sufficient for the minimization to consider only the large-scale components of the gradient of the cost function and, second, can the evolution of these large scale components be correctly predicted by a simplified model? Fig.3 from Laroche and Gauthier (1999) summarizes results from several experiments carried out with a simple barotropic model on a $\beta$-plane. The experiments were cast as identical twins with observations generated from a reference trajectory provided by the assimilating model and random noise representative of observation error was added to those synthetic observations. No background term is included. Fig. 3 shows the correla- 


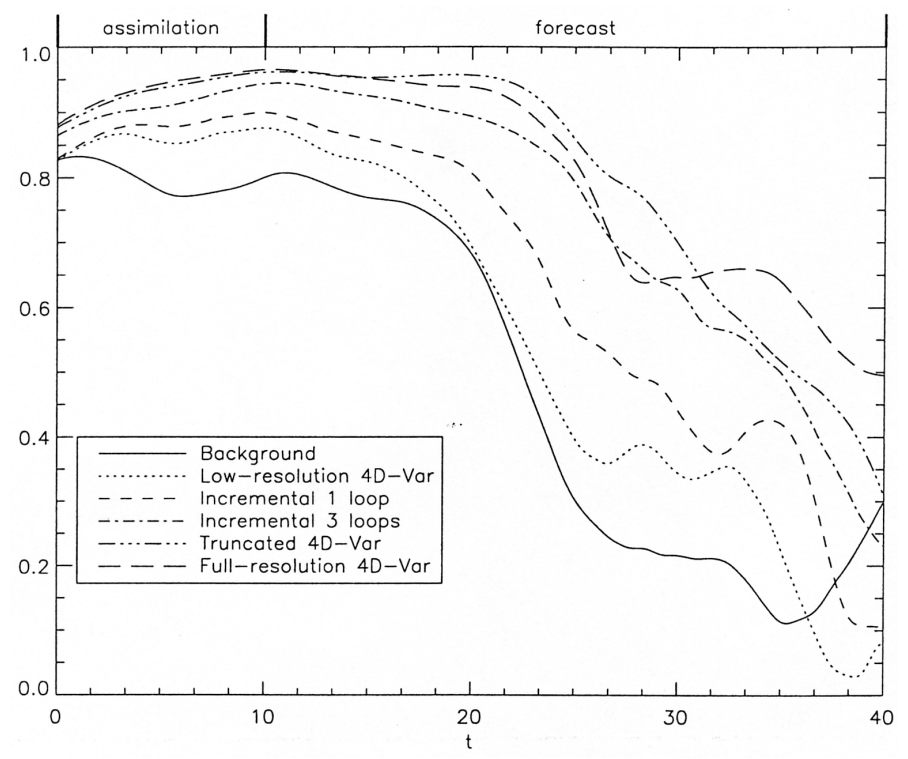

Figure 3. Correlation between the reference vorticity field and those obtained from various variational formulations. The synthetic observations are at low resolution and random noise has been added (from Laroche and Gauthier, 1999).

tion between the reference trajectory (or nature run) and the results from several experiments. The assimilation takes place over the first 10 nondimensional time units and forecasts at full resolution are made up to $t=40$. The full resolution $4 \mathrm{D}-\mathrm{V}$ ar shows the best that can be obtained from the low-resolution observations. Given the limit of predictability, a reasonable forecast can be obtained up to $t \cong 30$. The truncated 4D-Var experiment is one in which the adjoint model is used at the full resolution but the resulting gradient is truncated at a lower resolution (reduced by a factor of 4 ). In this case, Fig.3 shows that the truncated 4D-Var compares to the results obtained with the full resolution 4D-Var.

The low-resolution 4D-Var experiment is a complete 4D-Var based on the model at low-resolution. Fig. 3 indicates that it only marginally improves the fit to the reference trajectory compared to what is obtained from the starting point of the minimization. Finally, the incremental form of 4D-Var shows that with only one outer loop, the results are only slightly improved compared to the low-resolution 4D-Var. However, if three outer loops are considered, then the incremental formulation yields to a reasonable approximation of the truncated $4 \mathrm{D}-\mathrm{Var}$. These results show the crucial role of updating the trajectory by performing outer iterations of 4D-Var.

Another question raised by $4 \mathrm{D}-\mathrm{Var}$ is the impact of model error. It is implicitly assumed that any misfit to the observations is the result of error in the initial conditions. Experience shows that bad forecasts are often caused by errors in the model itself. An example is presented in the context of the barotropic model used in the experiments presented above. To mimic phase errors that occur with numerical weather prediction models that do not displace meteorological systems at the correct phase speed, synthetic 


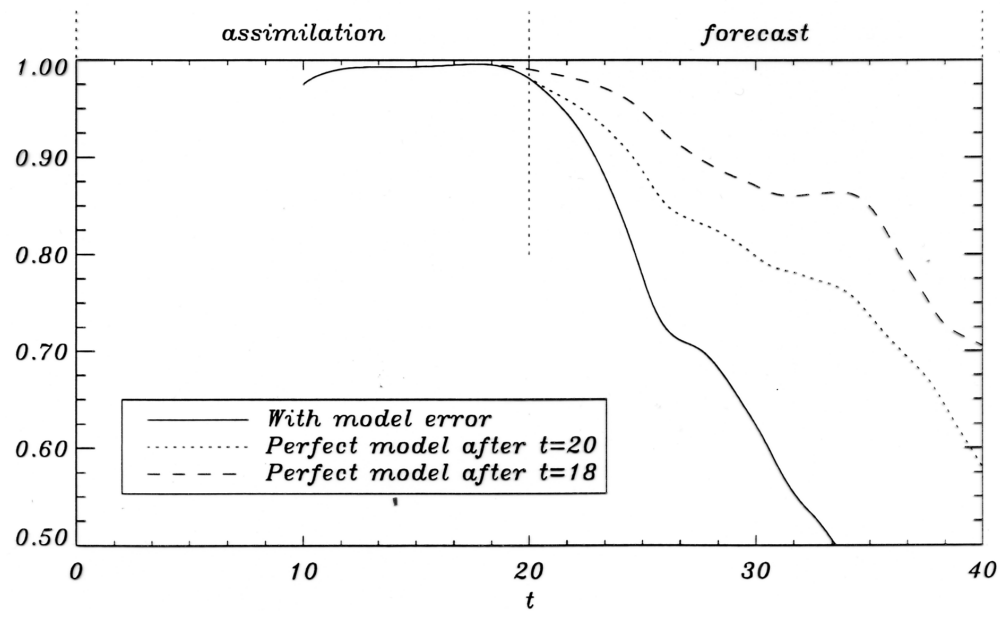

Figure 4. Solid line shows the correlation between the nature run obtained by using $\beta=0.5$ and the assimilation/forecast of a full 4D-Var based on a model using $\beta=0.4$. Using the perfect model to perform the forecast, the dashed (dotted) line show the correlation obtained when using the initial conditions at $\mathrm{t}=18(\mathrm{t}=20)$.

observations were generated from a nature run obtained with $\beta=0.5$ while $\beta$ was set to 0.4 in the model used in the assimilation. Fig. 4 shows the correlation between the true state and the assimilation/forecast based on the erroneous model: the assimilation window here is $10<\mathrm{t}<20$. It shows that the best solution is obtained not at the end of the assimilation window but at some earlier time. To test the quality of the analysis, the true model $(\beta=0.5)$ was used to make the forecast using the 4D-Var analysis at $\mathrm{t}=20$ (dotted line) and at $\mathrm{t}=18$ (dashed line) where the maximal correlation to the true state was obtained. It shows that the latter case yields to a substantially better forecast. So, even though these experiments used perfect observations at all grid points and at all times, the presence of model error cannot translate the information from the observations into a better forecast.

In operational systems, model error is often associated with weaknesses in the numerous physical parameterizations used in the model. The incremental formulation of $4 \mathrm{D}$-Var introduces a simplified set of physical parameterizations that should be consistent with those of the complete model. The development of a simplified set of physical parameterizations (deep convection, stratiform precipitation, surface and gravity-wave drag, vertical diffusion and radiation) is presented in Janisková et al. (1999) and Mahfouf (1999). As discussed in Mahfouf and Rabier (2000), this translated in a better fit to the observations within the inner loop of the 4D-Var. In Barkmeijer et al. (2001) and Puri et al. (2001), it is shown that the inclusion of a simplified physics in the TLM/Adj for the computation of the singular vectors used in their ensemble prediction system, has a significant impact on the spread of the resulting ensemble of forecasts.

Finally, it is important to stress that the 4D-Var analysis and forecast are better balanced with respect to the internal balance of the model. Fig.5 shows the average precipitation rates (over 24-h) as a function of forecast time. It clearly shows that, 


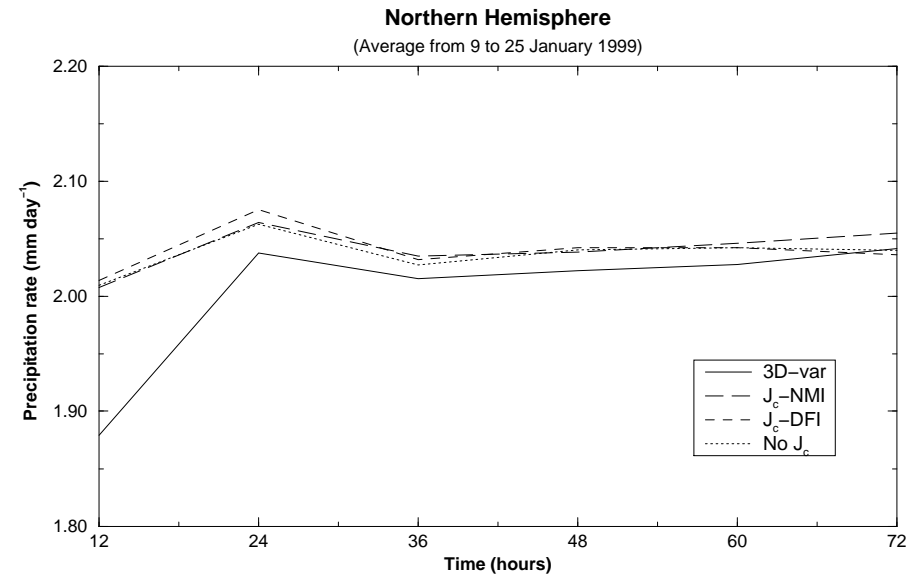

Figure 5. Average precipitation rates (in $\mathrm{mm} / \mathrm{day}$ ) as a function of forecast time. Those have been averaged over the Northern hemisphere for the two-week period extending from 9 to 25 January 1999. (see Gauthier and Thépaut, 2001 for details)

compared to 3D-Var, the 4D-Var analyses do not show a significant imbalance in the first hours of the forecast. This spin-up process is often associated with the presence of spurious gravity waves that need to be removed by an initialization process such as nonlinear normal mode initialization or a digital filter. The experiments of Gauthier and Thépaut (2001) show that even without any constraint to suppress these fast oscillations (No $\mathrm{J}_{\mathrm{c}}$ experiment), the 4D-Var analysis does not lead to an appreciable precipitation spin-up at the initial time.

\section{Experimentation with $4 \mathrm{D}-\mathrm{Var}$}

The introduction of the time dimension in the assimilation allows information to be extracted from a time series of observations. For instance, the 4D-Var assimilation of measurements related to passive tracers provides information about the winds (Andersson et al., 1994). Moreover, the 4D-Var analysis increments have a baroclinic structure that can be related to the fastest growing perturbations (Thépaut et al., 1996)., Based on the extensive experimentation carried out at ECMWF in preparation for the implementation of 4D-Var, Rabier et al. $(1998,2000)$ report that the main differences between the 3D-Var and 4D-Var analysis increments were observed in those regions identified as the most sensitive to perturbations in the initial conditions. The sensitive regions were determined from the singular vectors. Their results also indicate that the gain obtained with $4 \mathrm{D}$-Var lies in reducing the number of missed forecasts due to rapid cyclogenesis. This indicates that $4 \mathrm{D}$-Var is able to determine the changes to the initial conditions that will trigger or not the development of synoptic systems.

The advantage of $4 \mathrm{D}$-Var over $3 \mathrm{D}-\mathrm{V}$ ar is that it makes it possible to assimilate data at the observation time, which results in an increase of the volume of data that can be 
assimilated. Moreover, information contained in the temporal variation can also be extracted. However, Järvinen et al. (1999) present some difficulties, encountered in the assimilation of surface pressure data from stations reporting every hour. In regions where the real orography differs from that of the model, a bias can be introduced in the surface pressure data. When all hourly reports are assimilated, this results in a significant negative impact. This problem is more acute for isolated stations since there are no surrounding data to weigh against this biased estimate. Removing the effect of this bias from the data can be addressed in different ways and Järvinen et al. (1999) introduced a time-correlation in the observation error that manages to alleviate the problem. The net effect is to focus the analysis more on the variation of surface pressure than on the mean value of surface pressure. It is the surface pressure tendency that is more closely linked to developing baroclinic systems (Bengtsson, 1980).

\section{Conclusion}

Any variational problem raises the issue of the convergence of the minimization. One iteration of the minimization being particularly expensive in 4D-Var, its practical implementation has to limit the number of iterations to a rather low number, typically less than a hundred. Fisher and Andersson (2001) proposed a preconditioning of the minimization by approximating the Hessian of the 4D-Var cost function and this significantly improved the convergence. Problems in the conditioning could also explain a case of poor convergence in a 4D-Var experiment reported by Andersson et al. (2000). Convergence of the minimization is therefore still an issue and given the nonlinear nature of the model, so is the existence of multiple minima (Tanguay et al., 1995).

Currently, a lot of research is going on to address the importance of model error in 4D-Var and data assimilation in general. In particular, in the context of 4D-Var, the model is assumed to be perfect and even more, in the incremental formulation, the simplified model should be a good approximation of the more accurate high-resolution model with sophisticated physical parameterizations. As discussed in Bouttier (2001), extending the assimilation window to 12-h has accentuated the importance of these differences that could be diagnosed from the differences between observation departures computed with respect to the simplified and high-resolution models. Extending 4D-Var to longer assimilation windows may be more difficult than was thought initially due to the importance of model errors.

It was mentioned briefly that cycling 4D-Var requires that the background-error covariances reflect their flow-dependent nature. Results presented by Fisher and Andersson (2001) indicate that there is a long way from theory to practice. The reduced-rank Kalman filter used in their experiments provided a flow-dependent estimate of the covariances but this only had a marginal impact on the resulting forecasts.

Other avenues are being explored in 4D data assimilation. In particular, the ensemble Kalman filter (EnKF) (Evensen, 1997; Houtekamer and Mitchell, 2000) has been proposed recently to obtain a practical way of implementing a Kalman filter for complex models without having to develop the adjoint of a numerical model. The 
Monte-Carlo approach that supports the EnKF then raises some questions about the required size of the ensemble. Up to now, the direct estimate is often noisy and some assumptions must be introduced to address the rank deficiency problem. It also makes it more difficult to introduce non-Gaussian error statistics for errors, and to maintain dynamical balance in the analysis increments.

The 4D-Var algorithm imposes the model as a strong constraint, which has some limitations. Bennett and Thorburn (1992) introduced the weak constraint formulation in which the model is imposed only as a weak constraint. Because it uses the complete model trajectory and not only the initial conditions, this approach is much more demanding than 4D-Var. Recently, Xu and Daley (2002) introduced the accelerated representer algorithm which is referred to as 4D-PSAS in Courtier (1997). It corresponds to the dual formulation of 4D-Var and can be built from the same basic operators (e.g., model integrations, observation operators, and their tangent linear and adjoint, covariance models). In Lagarde et al. (2001), a graphical representation has been introduced to represent a wide class of assimilation algorithms altogether with their dual representations. Their analysis show that if some care is taken in developing a data assimilation system, it would be possible to reuse the same basic building blocks to obtain new algorithms. This would be an advantage for operational centres that must adapt quickly to new advances in a rapidly evolving field.

Acknowledgement. The author would like to thank Stéphane Laroche and Jean-Noël Thépaut for their comments on the manuscript.

\section{References}

Andersson, E., M. Fisher, R. Munro and A. McNally, 2000: Diagnosis of background errors for radiances and other observable quantities in a variational data assimilation scheme, and the explanation of a case of poor convergence. Quart. J.R. Meteor. Soc., 126, 1455-1472.

, E., J. Pailleux, J.N. Thépaut, J.R. Eyre, A.P. McNally, G.A. Kelly and P. Courtier, 1994: Use of cloud-cleared radiances in 3D and 4D variational data assimilation. Quart. J.R. Meteor. Soc., 120, 627653.

Barkmeijer, J., R. Buizza, T.N. Palmer, K. Puri and J.F. Mahfouf, 2001: Tropical singular vectors computed with linearized diabatic physics. Quart. J.R. Meteor. Soc., 127, 685-708.

Bengtsson, L., 1980: On the use of a time sequence of surface pressures in four-dimensional data assimilation. Tellus, 32, 189-197.

Bennett, A.F. and M.A. Thorburn, 1992: The generalized inverse of a nonlinear quasigeotrophic ocean circulation model. J. Phys. Ocean., 22, 213-230.

Bouttier, F., 2001: The development of 12-hourly 4D-Var. ECMWF Tech. Memo., No.348, 21 pages.

Cohn, S., A. da Silva, J. Guo, M. Sienkiewicz and D. Lamich 1998: Assessing the effects of data selection in the DAO Physical-Space Statistical Analysis System. Mon. Wea. Rev., 126, 2913-2926.

Courtier, P., E. Andersson, W. Heckley, J. Pailleux, D. Vasiljevic, M. Hamrud, A. Hollingsworth, F. Rabier and M. Fisher, 1998: The ECMWF implementation of three dimensional variational assimilation (3DVar). Part I: formulation. Quart. J.R. Meteor. Soc., 124, 1783-1808.

, 1997: Dual formulation of four-dimensional variational data assimilation. Quart. J.R. Meteor. Soc., 123, 2449-2461.

, J.N. Thépaut and A. Hollingsworth, 1994: A strategy for operational implementation of 4D-Var, using an incremental approach. Quart. J.R. Meteor. Soc., 120, 1367-1387.

Daley, R. and E. Barker, 2000: NAVDAS Source Book: NRL atmospheric variational data assimilation system. Naval Research Laboratory, Monterey, U.S.A., 151 pages. 
Desroziers, G., G. Hello and J.-N. Thépaut, 2003: A 4D-Var reanalysis of the FASTEX experiment. (to appear in Quart. J.R. Meteor. Soc.)

Evensen, G., 1997: Advanced data assimilation for strongly nonlinear dynamics. Mon. Wea. Rev., 125, 13421354.

Eyre, J.R., 1989: Inversion of cloudy satellite sounding radiances by nonlinear optimal estimation. I: Theory and simulation of TOVS. Quart. J.R. Meteor. Soc., 115, 1001-1026.

Fisher, M. and E. Andersson, 2001: Developments in 4D-Var and Kalman filtering. ECMWF Tech. Memorandum, No.347, 36 pages.

Gauthier, P., C. Charette, L. Fillion, P. Koclas and S. Laroche, 1999: Implementation of a 3D variational data assimilation system at the Canadian Meteorological Centre. Part I: The global analysis. AtmosphereOcean, 37, 103-156.

and J.-N. Thépaut, 2001: Impact of the digital filter as a weak constraint in the preoperational 4DVAR assimilation system of Météo-France. Mon. Wea. Rev., 129, 2089-2102.

Houtekamer, P.L. and H.L. Mitchell, 2001: A sequential ensemble Kalman filter for atmospheric data assimilation. Mon. Wea. Rev., 129, 123-137.

Janisková, M., J.-N. Thépaut and J.-F. Geleyn, 1999: Simplified and regular physical parameterizations for incremental four-dimensional variational assimilation. Mon. Wea. Rev., 127, $26-45$.

Järvinen, H., E. Andersson and F. Bouttier, 1999: Variational assimilation of time sequences of surface observations with serially correlated errors. Tellus, 51A, 468-487.

Lagarde, T., A. Piacentini and O. Thual, 2001: A new representation of data assimilation methods: the PALM flow-charting approach. Quart. J.R. Meteor. Soc., 127, 189-207.

Laroche, S., P. Gauthier, J. St-James and J. Morneau, 1999: Implementation of a 3D variational data assimilation system at the Canadian Meteorological Centre. Part II: the regional analysis. AtmosphereOcean, 37, 281-307.

and P. Gauthier, 1999: A validation of the incremental formulation of 4D variational data assimilation in a nonlinear barotropic flow. Tellus, 50A, 557-572.

Lorenc, A.C., S. P. Ballard, R. S. Bell, N. B. Ingleby, P. L. F. Andrews, D. M. Barker, J. R. Bray, A. M Clayton, T. Dalby, D. Li, T. J. Payne and F. W. Saunders, 2000: The Met. Office Global threedimensional variational data assimilation scheme. Quart. J.R. Meteor. Soc., 126, 2991-3012. 1194.

Mahfouf, J.F., 1999: Influence of physical processes on the tangent-linear approximation. Tellus, 51A, 147166.

- and F. Rabier, 2000: The ECMWF operational implementation of four dimensional variational assimilation. Part II: experimental results with improved physics. Quart. J.R. Meteor. Soc., 126, 11711190.

Parrish, D.F., and J.C. Derber, 1992: The National Meteorological Center's spectral statistical interpolation analysis system. Mon. Wea. Rev., 120, 1747-1763.

Puri, K., J. Barkmeijer and T.N. Palmer, 2001: Ensemble prediction of tropical cyclones using targeted diabatic singular vectors. Quart. J.R. Meteor. Soc., 127, 709-734

Rabier, F., J.-N. Thépaut and P. Courtier, 1998: Extended assimilation and forecast experiments with a fourdimensional variational assimilation system. Quart. J.R. Meteor. Soc., 124, 1861-1887.

—, H. Järvinen, E. Klinker, J.-F. Mahfouf and A. Simmons, 2000: The ECMWF operational implementation of four dimensional variational assimilation. Part I: experimental results with simplified physics. Quart. J.R. Meteor. Soc., 126, 1143-1170.

Talagrand O. and P. Courtier, 1987: Variational assimilation of meteorological observations with the adjoint vorticity equation. I. Theory. Quart. J. R. Met. Soc., 113, 1311-1328.

Tanguay, M., P. Bartello and P. Gauthier, 1995: Four-dimensional data assimilation with a wide range of scales. Tellus, 47A, 974-997.

Thépaut, J.-N. and P. Courtier, 1991: Four-dimensional data assimilation using the adjoint of a multilevel primitive equation model. Quart. J.R. Meteor. Soc., 117, 1225-1254.

, P. Courtier, G. Belaud and G. Lemaître, 1996: Dynamical structure functions in a four-dimensional variational assimilation: a case study. Quart. J. R. Met. Soc. , 122, 535-561.

$\mathrm{Xu}$, L. and R. Daley, 2002: Data assimilation with a barotropically unstable shallow water system using representer algorithms. Tellus, 54A, 125-137. 
Index

adjoint model,4

baroclinic structure, 7

digital filter, 7

innovations, 4

Kalman filter

ensemble Kalman filter, 8

reduced-rank, 8

model error,5, 8

Observation error

time-correlation, 8

preconditioning, 8

Representer accelerated representer algorithm (4D-PSAS), 9

Spin-up, 7

Tangent linear model,4

truncated 4D-Var,5

Variational assimilation 3D-Var,3

incremental 3D-Var,2

incremental 4D-Var,4

weak constraint formulation of 4DVar,9 